\title{
The Small Heat Shock Protein Cage from Methanococcus jannaschii is a Versatile Nano-scale Platform for Genetic and Chemical Modification
}

\author{
Michelle L. Flenniken, Deborah A. Willits, Susan Brumfield, \\ Mark J. Young, and Trevor Douglas
}

\section{Supporting Information: Figure 1}
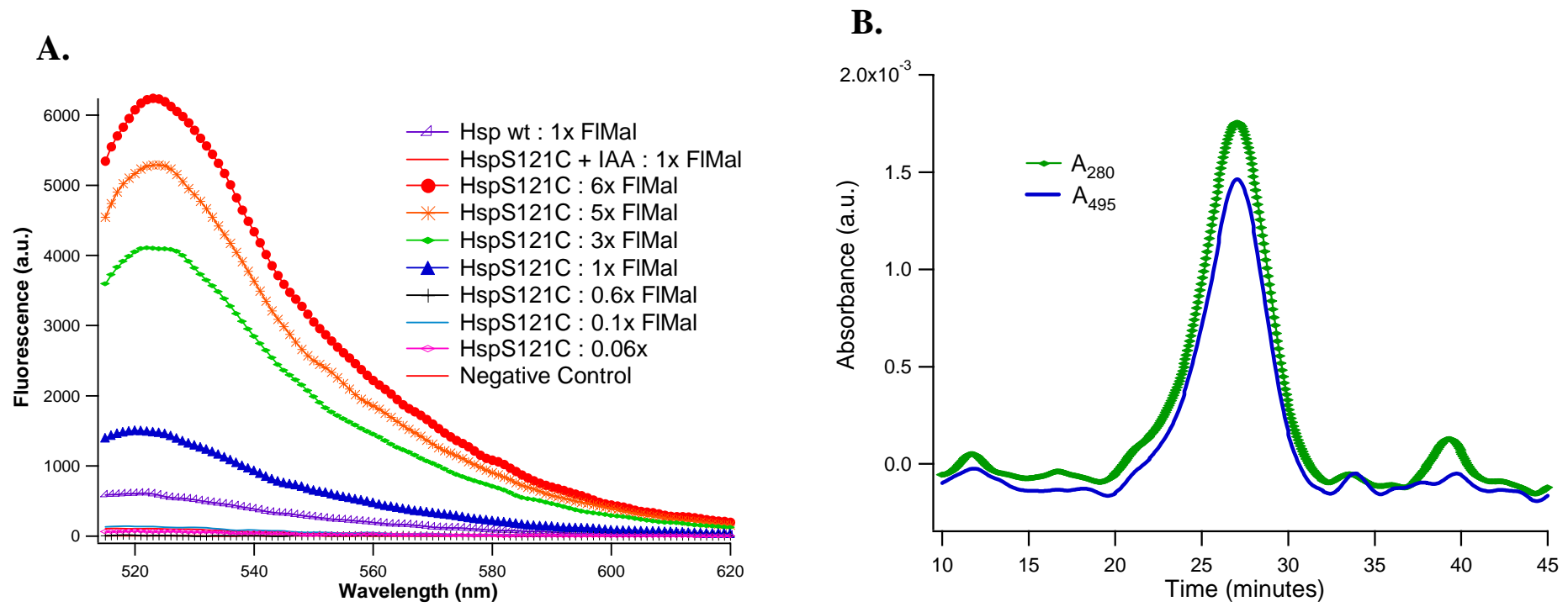

Supporting Information Figure 1. A. Fluorescence emmision of the HspS121C cages (either wild type (wt) or S121C) labeled with fluorescein-5-maleimide (FlMal). The fluorophore quantities listed are in molar equivalents of fluorophore per subunit. Protein free controls were performed at all fluorophore concentrations, one example is shown above (negative control $=10 x$ fluorophore only). In each case, no free fluorophore was detected by fluorescence. B. Size exclusion chromatography elution profile of HspS121C reacted with a 28 fold (28x) molar excess of fluorescein-5-maleimide per subunit. The profile illustrates the co-elution of fluorescein $\left(\mathrm{A}_{495} \mathrm{~nm}\right)$ and Hsp protein cage $\left(\mathrm{A}_{280} \mathrm{~nm}\right)$. 
The Small Heat Shock Protein Cage from Methanococcus jannaschii is a

Versatile Nano-scale Platform for Genetic and Chemical Modification

Michelle L. Flenniken, Deborah A. Willits, Susan Brumfield, Mark J. Young, and Trevor Douglas

Supporting Information - Figure 2 DYNAMIC LIGHT SCATTERING (DLS) DATA

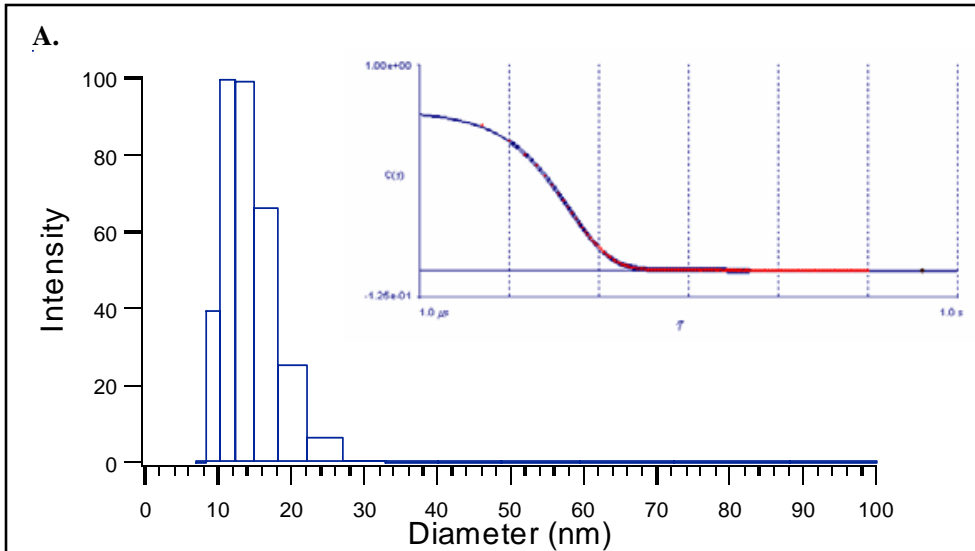

B.

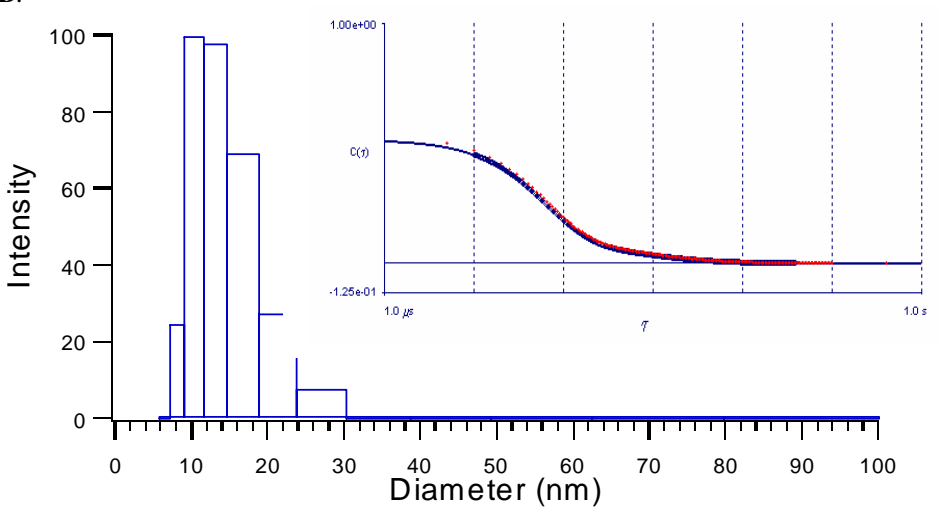

C.

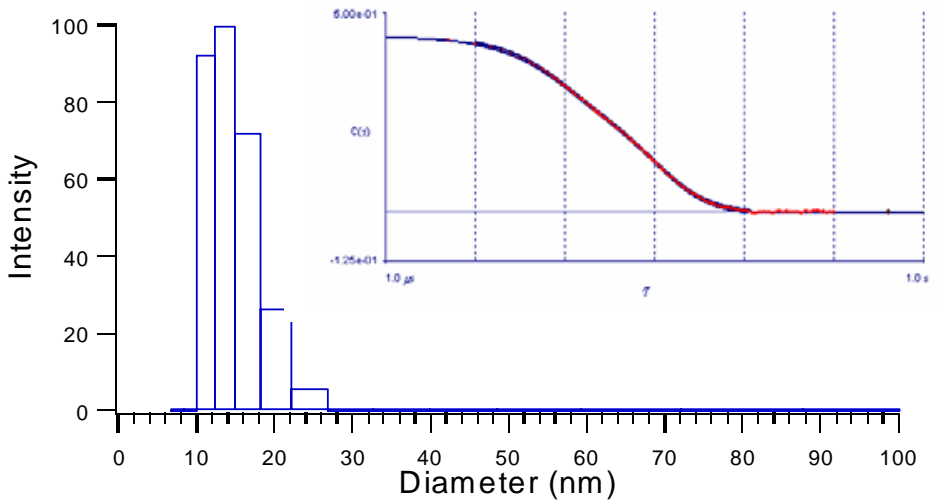

D.

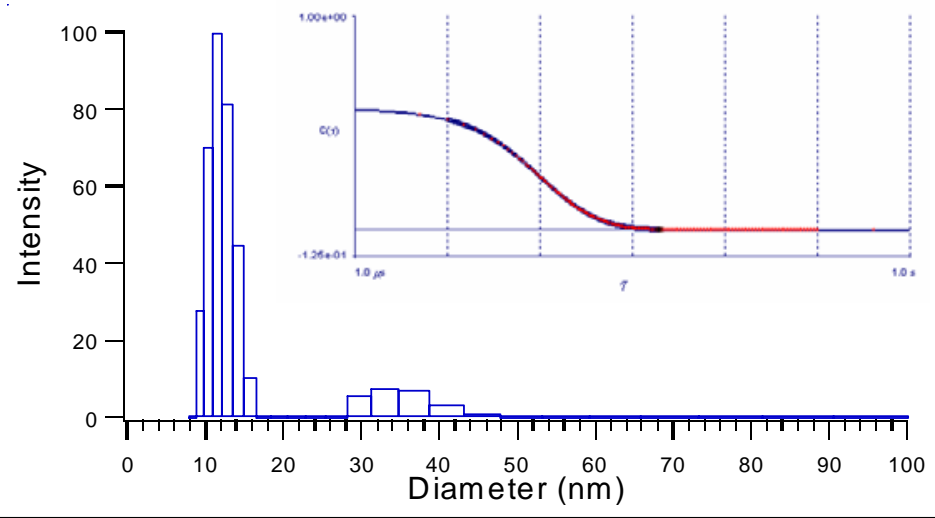

Supporting Information Figure 2. Dynamic Light Scattering (DLS) Data

A. Hsp G41C no experiment, Mean Diameter $=12.2 \mathrm{~nm}$

B. Hsp G41C FlMal labeled, Mean Diameter $=12.6 \mathrm{~nm}$

C. Hsp Wt 5(6-)FAM labeled, Mean Diameter $=12.1 \mathrm{~nm}$

D. Hsp G41C with Iron Oxide Core, Mean Diameter $=12.9 \mathrm{~nm}$ 
The Small Heat Shock Protein Cage from Methanococcus jannaschii is a Versatile Nano-scale Platform for Genetic and Chemical Modification

Michelle L. Flenniken, Deborah A. Willits, Susan Brumfield, Mark J. Young, and Trevor Douglas

\section{Supporting Information - Figure 3}

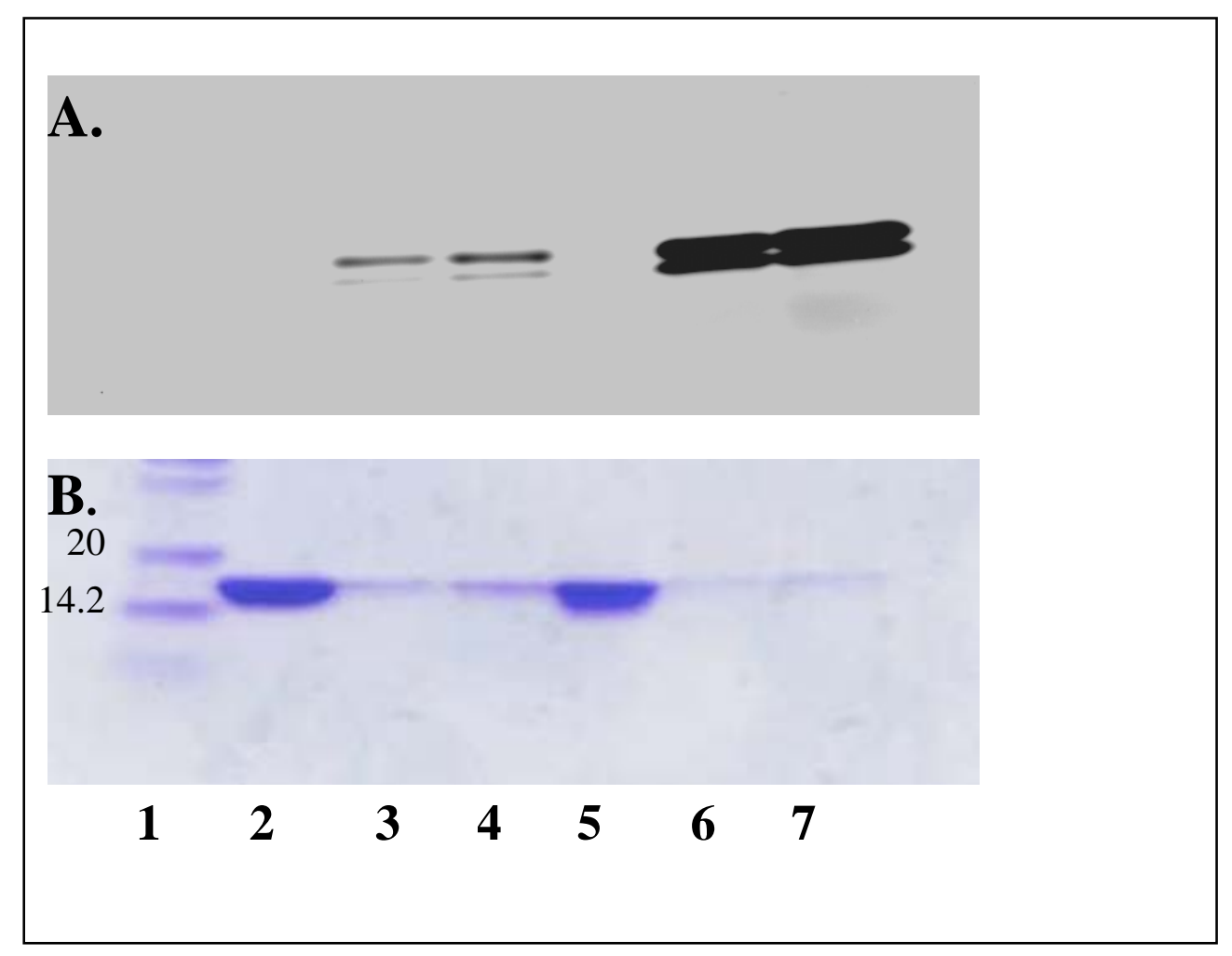

Supporting Information Figure 3. SDS-Polyacrylamide Gel Electrophoresis of Hsp The same gel is visualized first by (A.) fluorescence (inverted image) (Molecular Imager FX, BioRad) and subsequently by (B.) Coomassie Brilliant Blue staining. The lanes corresponding to the following samples are numbered below the gels: Lane $\mathbf{1}=$ Molecular weight ladder ( $\mathrm{kDa}$ ), Lane 2 = wtHsp only, Lanes 3\&4 = wtHsp Fl-Mal reaction, Lane 5 = HspG41C only, Lanes 6\&7 = HspG41C labeled with Fl-Mal. The gels show the covalent association of fluorescein-5-maleimide (Fl-Mal) with HspG41C; there is little association of fluorophore with the wtHsp cage. The lower band of the doublet (2 bands very close to each other) visualized by fluorescence imaging (A.) was confirmed to be an Hsp degradation product by mass spectrometry. 